\title{
Sobre la Formación del Docente-Facilitador de Personas Mayores
}

\author{
Enrique Fernández Lópiz' \\ 'Universidad de Granada - España
}

RESUMEN - Sobre la Formación del Docente-Facilitador de Personas Mayores. En este artículo se formula una propuesta de formación destinada a los docentes-facilitadores de actividades educativas con personas mayores. En el plano teórico se proponen ideas provenientes del campo de la Psicogerontología y la psicología dinámica. Este marco propone un modelo educativo participativo y un trato docente que promueva la autonomía del alumnado mayor. El modelo de formación se basa en la aplicación del Análisis Transaccional, cuyos objetivos principales se dirigen a la formación psicológica para el análisis de comportamientos docentes con mayores y la comunicación en el aula.

Palabras clave: Psicogerontología. Educación de Mayores. Análisis Transaccional.

ABSTRACT - Training of Teaching Staff for Older Adults. The present article is a proposal for the training of the teaching staff in charge of older adults education. On the theoretical aspect, we incorporated some ideas originating from the psychogerontology and the dynamic psychology. This theoretical, referential context has, in its favor, a relationship where it strongly presents a participating educational pattern and a teaching method that promote the autonomy of the older adult student. The proposed training pattern is based on the application of the Transactional Analysis to the teaching staff, whose main goals are to educate them in the analysis of teacher's behavior with older students and communication in the classroom.

Keywords: Psychogerontology. Elderly Education. Transactional Analysis.

Educação \& Realidade, Porto Alegre, v. 40, n. 1, p. 149-171, jan./mar. 2015.

149

Disponível em: <http://www.ufrgs.br/edu_realidade> 
Sobre la Formación del Docente-Facilitador de Personas Mayores

\section{La Educación con Personas de Edad Avanzada: el papel del educador}

La investigación y análisis sobre el perfil, las características y la formación de los educadores de adultos mayores son casi inexistentes en la literatura especializada; apenas se habla de una Gerontología Educativa. No es fácil encontrar estudios sobre aspectos motivacionales, perfiles profesionales o niveles de capacitación y de entrenamiento para realizar la tarea de educador con personas de edad. Esta carencia puede atribuirse al énfasis que los investigadores del proceso de enseñanza-aprendizaje en la madurez han otorgado a los mecanismos de adquisición del sujeto mayor, desatendiendo las características del contexto educativo y el rol del facilitador en las interacciones que promueven los aprendizajes y el desarrollo personal de este tipo de alumnado.

No obstante, existen algunas modalidades de organización pedagógica en este sentido, pero presentan una notable heterogeneidad. Si se las clasifica en relación al nivel de participación de los mayores en el diseño, gestión y ejecución de tales actividades, se encuentran dos modelos claramente diferenciados. Por una parte, el modelo de autogestión pedagógica, de mayor implantación en los países anglosajones y, por otra, el modelo científico-académico desarrollado en Francia y extendido por otros países europeos y latinoamericanos. En el primero, el educador-facilitador es un miembro del mismo grupo de edad adulta mayor que coordina las actividades de autoaprendizaje que el grupo se plantea como meta. En el segundo, el docente-facilitador pertenece a una institución académica y comparte su conocimiento con el grupo de mayores, introduciéndolos en un campo disciplinar, transfiriendo conocimientos y contribuyendo al desarrollo de destrezas para que los alumnos construyan su propio saber sobre un área o materia.

El enfoque general desde el que se plantea la actividad educativa va a determinar no sólo la posición del facilitador, sino también el tipo de competencias profesionales que este debe poseer para ser eficaz en su trabajo. Algunas organizaciones educativas sostienen la conveniencia de que los facilitadores sean del mismo grupo generacional que los participantes, pues comparten experiencias vitales similares y están en mejores condiciones de comprenderlos. En otras instituciones se prefieren profesores más jóvenes, arguyéndose su alta motivación para trabajar y una disposición a innovar las formas tradicionales de enseñanza.

Por nuestra experiencia sabemos que el facilitador juega un papel relevante en el aprendizaje y la motivación de los participantes. En investigaciones que hemos dirigido (Yuni, 1999) comprobamos que más de la mitad del alumnado mayor preguntado al respecto, atribuye sus aprendizajes al trabajo del profesor, identificando tres aspectos fundamentales en la tarea de estos:

- Capacidad del profesorado para seleccionar contenidos significativos 
- Competencia para favorecer un clima adecuado de participación, expresión y vínculos entre el alumnado;

- Autoridad como referente intelectual y ético del grupo.

Estos datos revelan que el facilitador de adultos mayores es un personaje principal y en absoluto accesorio en la tarea educativa con esta población de edad. Es el principal responsable del aprendizaje, de favorecer una participación eficaz y de preparar situaciones en las que el alumnado pueda apropiarse progresivamente del conocimiento, así como de evaluar las adquisiciones realizadas por los sujetos. Pero a la vez, es también el garante de la puesta en escena de otras dimensiones socio-afectivas en el aula.

\section{Determinantes de la Función Docente con Alumnado Mayor}

La eficacia de la actuación educativa del docente-facilitador se relaciona a nuestro juicio con los siguientes aspectos (Fernández Lópiz, 2002, p. 176).

- Filosofía educativa y los valores que sustentan la práctica;

- Concepción positiva acerca de las posibilidades de desarrollo intelectual, personal y social de las personas mayores;

- Capacidad para superar los prejuicios culturales anti-mayor (“viejismo”) (Fernández Lópiz, 1997; 2003b);

- Posibilidad de establecer un juego educativo que potencie las potencialidades, al decir de Vygotski (Zona de Desarrollo Próximo), mediante modalidades interactivas de aprendizaje;

- Capacidad para trabajar con individuos con altos niveles de individuación y de experiencia personal, incluyendo la variable género (Fernández Lópiz; Marín Parra; Alfaro Llaneza, 1999).

Los aspectos señalados hunden sus raíces en las concepciones actuales de la Psicogerontología y en la perspectiva pedagógica y filosófica que fundamenta la educación de las personas de edad (Fernández Lópiz, 2013; Bedmar; Fresneda; Muñoz, 2004; Martín García, 1994).

En lo que sigue fundamentaremos un modelo formativo destinado a los facilitadores, e incluimos algunas reflexiones sobre el grupo educativo.

\section{Concepciones Básicas desde la Psicogerontología para Educadores de Mayores}

Estas concepciones básicas que ahora exponemos quieren contribuir a la definición de una manera de actuación educativa con adultos mayores. Nos inspiramos en una concepción donde el educador sirve de mediador para el fomento de las posibilidades de expresión del ma-

Educação \& Realidade, Porto Alegre, v. 40, n. 1, p. 149-171, jan./mar. 2015.

Disponível em: <http://www.ufrgs.br/edu_realidade> 
yor; para su inserción dentro de los espacios sociales y para dignificar la vejez como polo de la existencia en el que la vida tiene una posibilidad abierta de futuro.

Para perfilar el rol del educador de mayores hay que conocer las características que definen las competencias de este alumnado en sus diferentes dimensiones biológica, cognitiva y afectivo-social (Fernández Lópiz, 1998a; 2001; Bedmar; Fresneda; Muñoz, 2004).

Los estudios actuales sobre el desarrollo en la vejez se basan en la psicología del Ciclo Vital. La idea es que los mayores son personas que han vivido una significativa carga de experiencias personales, sociales, históricas y culturales incorporadas a su mismidad, que tienen una enorme importancia en el desarrollo de este grupo de edad. Así, los educadores-facilitadores deben entender que su alumnado se ha "hecho" desde una íntima relación prolongada con la vida y con la Historia. La condición de "persona mayor" viene impuesta en gran medida por la sociedad y la cultura en la que ha vivido, y esas son sus referencias. Si la vejez - según Simone de Beauvoir (1983) - como destino biológico, es una realidad transhistórica, ese destino es vivido de manera variable según el contexto social; o sea, el significado de la vejez en el seno de una sociedad, hace que a través de ella se entienda la dimensión histórica de la misma. O sea, la biología puede declinar, pero el espíritu, en lo social y humano, ascender.

Hay algunos señalados aspectos para subrayar, a fin de que quienes se vayan a dedicar a esta interesante labor las consideren y reflexionen (Fernández Lópiz, 2003b).

- Cambio. Una primera idea es que la posibilidad de cambio es consustancial a la vida en todo su curso, incluida la tercera edad.

- Pérdidas y ganancias. Tampoco se mantiene hoy día la idea del deterioro en los mayores, pues el curso del desarrollo humano está constituido siempre por la ocurrencia de ganancias (crecimiento) y pérdidas (declive). El educador ha de promocionar el enriquecimiento y favorecer la consecución de lo que autores como Baltes y Smith (1994) han denominado "envejecimiento dichoso".

- Multidireccionalidad. Explica las diversas direcciones que adopta el desarrollo humano a lo largo de la vida. En la vejez las cualidades que declinan más son las más canalizadas biológicamente, en tanto que las derivadas del proceso de socialización, enculturización y experiencia "cristalizan" en nuevas posibilidades que trascienden la biología, tal el caso de la sabiduría (Fernández Lópiz, 2000a, p. 99-104).

- Heterogeneidad. Los mayores difieren mucho entre sí, de manera que se debe ser consciente de que el alumnado mayor es más heterogéneo que si de jóvenes se tratara (Burba; Fernández Lópiz; González Verheust; Luque, 2013). 
- Historicidad. La persona mayor es un "sujeto histórico" pues las condiciones sociales y económicas vividas determinan en gran medida el curso del desarrollo en esta edad.

- No normatividad. El educador debe saber que no hay una etapa uniforme que delinea la edad mayor de una manera exacta.

- Educación y optimización. Finalmente, diremos que los esfuerzos en la prevención y corrección de los aspectos evolutivos son un punto de partida que alzaprima el valor de la educación como elemento saludable en un sentido amplio.

\section{Plasticidad Cognitiva}

En las investigaciones sobre "potenciales de aprendizaje" en adultos mayores se ha demostrado la mejora en habilidades de inteligencia, tras diferentes formas de entrenamiento (Calero; Lozano, 1994, p. 97). También, los programas de entrenamiento en tareas de memoria han conseguido resultados positivos (Fernández-Ballesteros et al., 1992). Asimismo, arrojan buenos resultados los programas de intervención psicoeducativa y las intervenciones en actividades de la vida diaria, que han permitido consolidar el concepto de "plasticidad cognitiva", entendida como la posibilidad de adquirir o readquirir información a través de estrategias de enseñanza y manipulación ambiental. Las habilidades interpersonales también han podido ser optimizadas significativamente en personas mayores que presentaban dificultades en esta esfera.

Consiguientemente, son susceptibles de entrenamiento prácticamente todas las habilidades intelectuales, tanto las de orden cotidiano, como las más académicas o intelectuales propiamente dichas, sin olvidar el terreno socio-afectivo. En este sentido los efectos de la educación son muy importantes, tanto para la población mayor con escolaridad alta, como para la de media o baja escolarización (Yuni, 1999).

\section{Paradigmas Educativos con Mayores}

La educación permanente es una manera de potenciar las posibilidades de realización personal, así como la participación en la vida social de los alumnos mayores. Esto quiere decir que este grupo de edad necesita oportunidades para enfrentarse a los cambios de todo tipo que se están produciendo en nuestro entorno social y cultural. Y es justamente esta necesidad la que ilustra la preocupación de que los mayores puedan conocer y estar al día de dichas transformaciones y sentir así reforzada su seguridad y a la vez su propia identidad ante tan sustanciales cambios (Fernández Lópiz, 1998b).

La participación de sujetos mayores en la educación es un hecho constatable en diversos contextos y períodos históricos pasados. Sin embargo, a comienzos de los años setenta comienzan a desarrollarse

Educação \& Realidade, Porto Alegre, v. 40, n. 1, p. 149-171, jan./mar. 2015. 
Sobre la Formación del Docente-Facilitador de Personas Mayores

iniciativas que instituyen nuevas prácticas educativas, al trascender los objetivos usuales de alfabetización o post-alfabetización y adoptar una perspectiva marcadamente generacional. Varios autores ubican en este momento la aparición de un nuevo campo de estudio y de intervenciones pedagógica: la gerontología educativa (Martín García, 1994; Bedmar; Fresneda; Muñoz, 2004). La aparición de modalidades educativas innovadoras como la Open University en Inglaterra o las Universidades de la Tercera Edad en Toulouse (Francia), producen un entrecruzamiento entre la gerontología social y la pedagogía que sienta las bases para el posterior desarrollo de este campo.

Existen tres paradigmas que sustentan la educación y la gerontología (Fernández Lópiz, 2012a, p. 177-178).

a.- Paradigma funcionalista. Con una fuerte orientación pragmática, tiene una marcada influencia en la educación para la salud y en las microexperiencias educativas orientadas a favorecer el cambio de hábitos y de modificación del estilo de vida.

b.- Paradigma interpretativo. Interesado en los significados que los sujetos otorgan a su educación y que se vincula a autores pertenecientes a diversas corrientes del humanismo. Este paradigma subraya los procesos de realización personal en el marco de la búsqueda de sentido para la existencia.

c.- Paradigma vinculado a la pedagogía crítica de adultos. El paradigma crítico propone la necesidad de dar un lugar a las personas mayores dentro de la estructura socio-política. Por lo tanto, los mayores son los que han de diseñar y ocuparse de su educación, lo que implica que el rol de este grupo de edad debe ser analizado y considerado en profundidad y tener en cuenta la capacidad de empoderamiento en los mayores(empowerment): hacer efectiva su participación en los procesos de toma de decisiones y examinar las relaciones entre conocimiento, poder y control de parte del alumnado mayor.

En definitiva, la educación con mayores tiene una naturaleza tanto preventiva como compensatoria, que le confiere un carácter positivo que busca contribuir a que la persona mayor mejore y también que se conozca mejor. Por eso tiene un sesgo ideológico que persigue un mayor protagonismo de los mayores en la confección de actividades y programas educativos, a fin de ser ellos mismos, artífices y protagonistas.

\section{Competencia Intelectual y Vejez: sabiduría y envejecimiento dichoso}

En el estudio psicométrico suele distinguirse entre una inteligencia fluida y otra cristalizada. La inteligencia cristalizada, vinculada al funcionamiento neurobiológico y relacionada con la velocidad de respuesta, decae antes con la edad (habilidades vulnerables). La inteligencia cristalizada deriva de los procesos de enculturización, educativos y 
acumulación de experiencia y se mantiene con el tiempo (habilidades mantenidas) (Fernández Lópiz, 2002, p. 159-161).

Algunas autoras como Dittman-Kohli (1986), entienden que la llamada inteligencia cristalizada está unida al esquema de razonamiento verbal de tareas y a las capacidades intelectuales particulares de cada individuo. La autora ha preferido la utilización del término "inteligencia sintética o pragmática". Este tipo de inteligencia sintetizada sirve como un potencial de conocimiento, como la capacidad para resolver los problemas relacionados con las tareas propias de la vida. De cara a la tarea educativa, es preciso tener en cuenta que la inteligencia de las personas mayores es, como señala Dittman-Kohli (1986), un potencial con el que debemos trabajar, dado el carácter multidimensional de la misma (alberga una multiplicidad de habilidades que cabe actualizar), que además posee un importante margen de plasticidad.

La misma Dittman-Kohli (1986) y nosotros mismos (Fernández Lópiz, 2012a, p. 173-175), pensamos que la inteligencia de la persona mayor tiene varias cualidades que no debemos olvidar en educación:

a. La diferencia existente entre el potencial intelectual y el nivel de ejecución; o sea, la potencialidad puede ser enriquecida en la inmersión educativa favoreciendo la performance, haciendo que la persona mayor pueda hacer efectivas cualidades que tiene y que sólo precisan de reactivación y emergencia.

b. En la vejez hemos de considerar la importancia que sobre el curso de la inteligencia tiene la especialización individual adquirida con anterioridad, tanto en lo que concierne a capacitación académica o laboral, como en lo que se refiere a la propia experiencia vital. De hecho, la educación ha de servir también para el aprovechamiento de esos recursos de experiencia: profesionales de la medicina, la agricultura, la ingeniería, la filosofía, la mecánica, etc., que tienen una pericia en sus profesiones, pero también maestría en los asuntos de la existencia.

c. Sabiduría y "envejecimiento dichoso". Los recursos de conocimiento en la vejez son muy complejos. Tal es el caso de la sabiduría, que incluye: un conocimiento general y de procedimientos para la vida; contextualismo o capacidad de encuadre de los problemas de la vida; relativismo: interpretaciones y soluciones diferentes; incertidumbre: imprevisibilidad e indeterminación de la existencia; sensibilidad, sociabilidady discreción; perspicacia y sagacidad: intuición, sutileza, lucidez; buen humor; y en el plano personal y ético: autodesarrollo y autotrascendencia (psicoanálisis) (Fernández Lópiz, 2002, p. 138-143).

d. Lo que Clayton (1982) define como un tipo de inteligencia capaz de operar desde los principios de contradicción, paradoja y cambio. 
Sobre la Formación del Docente-Facilitador de Personas Mayores

e. Y según Sternberg (1985), el saber de las personas mayores es un tipo de conocimiento interpretativo que combina distintas dimensiones que conllevan una comprensión de los límites y valores de la vida y del vivir.

En resumen, la inteligencia en este grupo de edad, cara al hecho educativo, no concierne sólo a aspectos formales y descontextualizados del conocimiento, sino también a ese punto en que el conocimiento se vincula con el contexto sociocultural, con el autoconocimiento, con los asuntos de la vida; y habrá que tener en cuenta también, otras cualidades en que lo cognitivo, lo afectivo y la capacidad de reflexión encuentran un espacio común en cualidades como la introspección, la intuición, la comprensión, la delicadeza, la capacidad para aconsejar, el reconocimiento y mejor aceptación de la ambigüedad, de la complejidad y de la incertidumbre, la capacidad para identificar la verdadera dimensión de una cuestión ante las alternativas y las soluciones posibles, o el sentido común en áreas como la planificación, la dirección y la "revisión de la vida". Como apunta Eduardo Galeano en El Libro de los Abrazos: Sabios doctores de Ética y Moral han de ser los pescadores de la costa colombiana, que inventaron la palabra 'sentipensante' para definir al lenguaje que dice la verdad; el lenguaje, en definitiva, del hombre sabio.

Hemos traído a colación estas ideas para recalcar que los recursos de conocimiento de las personas mayores son algo más que un coeficiente o una valoración basada en tests. Es preciso insistir, cara al personal educador-facilitador, que tienen frente a ellos un alumnado tal vez más lento, más distráctil, menos despierto para tareas que exigen rapidez y premura; pero individuos que, en el plano del conocimiento, pueden llegar a ser muy creativos en relación al hallazgo y reformulación de problemas o la posibilidad de superpreguntar ante interrogantes imprecisas que se puedan tratar en el aula. Una forma de conocimiento y de ser inteligentes, en suma, mediada por nuevas indagaciones y pesquisas que implican una mayor capacidad de reflexión, de gusto por el saber, por ir a las cosas sustanciales e importantes, y la preferencia por indagaciones conceptuales novedosas. De hecho, la inquietud por el descubrimiento es un importante componente actitudinal del conocimiento y de la productividad creadora en el alumnado mayor, que sabe estar abierto a la preocupación, al saber y al conocer, que incluye la apertura a las cuestiones que se plantean en clase y la orientación hacia la innovación.

\section{La Motivación Educativa en Alumnado Mayor}

Basándonos en entrevistas clínicas realizadas a alumnado mayor, y tras realizar un análisis de la consistencia de las respuestas, hemos elaborado una serie de categorías conceptuales tomadas de la realidad educativa con este grupo de edad, que sirven para explicar qué empuja a los mayores a la educación, cuáles son las motivaciones académicas 
en esta edad. Lo que hicimos fue ordenar las respuestas ofrecidas por este colectivo a la pregunta de "por qué estaban estudiando en las aulas de mayores"; "qué les había impulsado a ello". Este apartado tiene mucho valor a la hora de que los educadores comprendan mejor las razones que empujan a los mayores a asistir a sus cursos y seminarios.

Según nuestras investigaciones, las motivaciones fueron las siguientes (Yuni, 1997; Fernández Lópiz, 2002, p. 172-175).

7.1. Motivación de curiosidad ilusoria. La caracterización de este tipo de motivación es la curiosidad y la necesidad de descubrimiento, y posee componentes de autorrealización intelectual y de superación personal. Subyace a este tipo motivacional la creencia de que la educación permite acceder a la cultura y que esta tiene un valor en sí misma que enriquece a quien puede apropiarse de ella. El objetivo de los sujetos que se conducen por este afán es educarsey satisfacer el deseo de conocimiento puramente. La característica ilusoria refleja las atribuciones que realizan los individuos en relación a la posibilidad de educarse y de progresar personalmente. Desde la teoría psicoanalítica (Winnicott, 1981루 Fernández Lópiz; Yuni, 1998), la educación es percibida como espacio intermedio que permite desarrollar la confianza en sí mismo y avanzar en el proceso de individuación. La educación es atractiva en tanto espacio «ilusionante» (por ejemplo, el gusto por la ciencia, el arte, etc.).

Las respuestas de los sujetos encuestados aplicables a esta categoría son del tipo: Aprender a realizarme como persona y enriquecer mi interior. Aún siento como una necesidad de poder agregar a mi vida una nueva esperanza. Buscar un nuevo espacio para llenar mis inquietudes. Cultivarme, estar con la gente, llenar un vacío. Enriquecerme como persona y también intelectualmente, etc.

7.2. Motivación de orientación pragmática. El acento está puesto en el aprendizaje de habilidades, conocimientos y técnicas específicas que permitan alcanzar determinadas metas. Su referencia es siempre la utilidad que tal adquisición puede brindar. Este tipo de motivación denota un carácter práctico del alumno que elige actividades que puedan servirle para alcanzar sus objetivos (por ejemplo, el aprendizaje de idiomas de cara a un viaje). Los sujetos se orientan a saberes específicos desde una percepción utilitarista del conocimiento: el objetivo es "aprender para...".

Respuestas tipo fueron: Curiosidad por los temas sobre calidad de vida. Inglés, por estimarlo indispensable en los viajes. Aprender calidad cocina. Aprender computación para comunicarme con mis nietos, etc.

7.3. Motivación de readaptación emocional. Está relacionada con las necesidades de adaptación que experimentan los sujetos frente a situaciones de estrés o de desajuste psicológico. Esta motivación representa una forma de progreso y readaptación, frente a determinadas crisis emocionales producidas por eventos dramáticos o difíciles comunes, tales como la viudez, la jubilación, una enfermedad, etc.

Educação \& Realidade, Porto Alegre, v. 40, n. 1, p. 149-171, jan./mar. 2015. 
Sobre la Formación del Docente-Facilitador de Personas Mayores

Respuestas del tipo: Tratar de salir adelante después de mi viudez. Decidí inscribirme por estar sola en mi casa y fue una experiencia sumamente enriquecedora. Después de una larga enfermedad decidí cambiar y vivir de otro modo. Una necesidad de hacer algo que cambiara el ritmo monótono y sin sentido de mi vida, etc.

7.4. Motivación de entrenamiento. El aprendizaje es entendido como entrenamiento preventivo para dilatar la funcionalidad o evitar el declive de los desempeños intelectuales y motores. Estos sujetos consideran que el mantenimiento de su buena forma es en gran parte responsabilidad suya, y atribuyen una considerable importancia a mantener la cabeza bien y a permanecer activos. Esta motivación se refiere a la necesidad de preservar un sentido de auto-eficacia y de continuidad en los niveles de desarrollo intelectual, físico y social.

Con respuestas tales como: Busqué tener una actividad que me mantenga activa física y mentalmente. Emplear mi tiempo de ocio, entrenar mi agotada memoria, aprender nuevos conocimientos. Conseguir que mi mente y mi inteligencia no se deterioraran. Hacer actividades de talleres y mantenerme activa física e intelectualmente. Aumentar mis relaciones sociales.

7.5. Motivación por ilusión diferida. Denota una orientación del sujeto a la actividad por el efecto de la presión que otras personas significativas realizan sobre él. Este tipo de motivación no se refiere a la ilusión por insertarse e incluirse en un espacio creativo y de desarrollo personal. Se trataría más bien de una inclinación producida por la identificación con algunos rasgos, valores o actitudes que han recibido de quienes les indujeron (cónyuge, amigos, vecinos, el médico, etc.).

Respuestas como: Porque me comunicaron que las clases eran muy interesantes y los profesores muy bien. Decidí inscribirme por invitaciones reiteradas de amigos con intención de asistir simplemente como oyente. Me inscribió mi hija para que me ocupara en algo. Por consejo médico.

7.6. Motivación de asignatura pendiente. Este tipo de motivación nos muestra claramente cómo hay metas y necesidades internas de los sujetos que se mantienen a través del tiempo. La educación es vista como compensatoria de lo que no se pudo hacer cuando joven $\mathrm{y}$, a la vez, es vista como concreción de lo esperado. Un componente importante de este tipo de motivación es que la posibilidad de aprender se da entre personas de la misma edad. Muestran un sentido de competencia para aprender y expresan su estímulo por realizar "el sueño de toda su vida".

Respuestas tales como: Deseaba saldar asignaturas pendientes ya que en su momento no seguí ninguna carrera universitaria. Porque yo quería aprender algo más en mi vida, ya que de joven no pude. Cumplimentar deseos no realizados anteriormente. Hacer algo que por miedo, timidez, ridículo o presiones familiares no lo pude hacer (teatro). 


\section{Buscando la Grupalidad en el Aula}

Durante décadas se pensó la educación como una actividad dirigida a niños y a jóvenes. En nuestro actual mundo, cada vez más envejecido, en que además las personas mayores se conservan saludables tras la jubilación y con necesidad de operar socialmente de forma activa, la educación dirigida a este grupo de edad, se ha revelado como un recurso social e institucional muy importante, para conseguir objetivos diversos en las legítimas pretensiones de los mayores para "añadir vida a los años”, como reza el conocido dicho. Más que una línea meramente instructiva, la educación con mayores ha de ser, desde nuestra consideración, saludable. El espacio educativo ha de tener el común denominador de combinar los aspectos teóricos, académicos o formativos, con aquellos otros de desarrollo personal, de socialización e inserción dentro del grupo. Es así que, entre educador-facilitador y alumnado, se ha de establecer una zona formativa en la que el alumnado, no sólo profundiza sobre los contenidos concretos de un seminario o taller, sino que además intercambia experiencias personales. Pero veamos esto más detenidamente.

Desde la conocida obra del gran psicólogo Kurt Lewin, sabemos que en todo contexto en el que aparecen diversos componentes, se da un determinado tipo de relaciones que generan unas "fuerzas de campo" que alteran las propiedades meramente individuales de sus componentes. La congregación educador-educandos es, al modo gestáltico, una "totalidad" en la que el conjunto es más que la suma de sus partes. Es decir, en la situación educativa, se dan unas circunstancias que modifican la relación individual que se establece entre el "facilitador" y los "facilitados", entre el educador y los educandos, y su objeto de estudio en el contexto del aula. En la medida que varias personas se reúnen en torno a un tema, aparecen unas circunstancias que lo modifican, tornándolo más complejo y también más rico.

Dado que los grupos propios de la educación con adultos mayores no deben ser muy numerosos (Naval, 2001), el objetivo grupal-educativo debe ser, desarrollar un trabajo en torno a una serie de capítulos o áreas de interés, aprovechando las condiciones que el discreto número de participantes facilita. En efecto, en la educación con mayores, el número de alumnos suele ser pequeño y de este modo, existe la oportunidad de generar pautas, no sólo para el progreso intelectual, sino para permitir que los componentes del seminario o taller participen de forma activa en el conocimiento interpersonal (Fernández Lópiz, 2000a). El educador puede adquirir, de esta forma, un conocimiento más preciso de los intereses y particularidades del alumnado, y este puede beneficiarse de las interacciones entre pares y de una mayor proximidad con el facilitador, lo que redunda en un general beneficio. Este trabajo pedagógico alrededor de una serie diversa de temas, también toca de lleno el mundo de los afectos: compartir experiencias personales, aumentar

Educação \& Realidade, Porto Alegre, v. 40, n. 1, p. 149-171, jan./mar. 2015.

Disponível em: <http://www.ufrgs.br/edu_realidade> 
Sobre la Formación del Docente-Facilitador de Personas Mayores

el grado de confianza, fomentar la cohesión, ayudar en las incorporaciones enriquecedoras, etc. En suma, la educación con mayores debe incluir también en su proceso los aspectos afectivos subyacentes a la tarea formativa. El educador ha de conformar un espacio grupal en el que el alumnado trate de profundizar sobre aspectos de su actual vivir, de su posición como individuos de edad en una atmósfera de libertad suficiente como para aceptar y tolerar libremente la discusión de ideas. Es, de este modo, una experiencia educativa y a la vez, terapéutica.

\section{La Constitución del Grupo Educativo}

Ya hemos referido la importancia que para la educación con mayores tiene el hecho de que sea el espacio de clase, un espacio grupal en el que poder compartir y participar en una especie de transunión. Cuando esto sucede, lo que se comparte no es sólo el material motivo de aprendizaje, sino también la fuerza motriz que arrastra esta labor formativa: los afectos, las filias y las fobias, los afanes, el drama personal que en cada sujeto mayor requiere del pretexto para ser rememorado, que necesita de ese tipo de memoria que llamamos en Psicogerontología "reminiscencia”, y que tiene la posibilidad de actualizarse en cada paso que facilitador y grupo dan. Y no decimos este extremo caprichosamente. En nuestras investigaciones y experiencias dirigidas y participadas, hemos constatado que los mayores asisten a los seminarios y talleres en una gran proporción por razones que tienen que ver con asuntos personales, la readaptación emocional ante eventos vitales (como la viudez, la enfermedad, la jubilación o la pérdida de seres queridos) y de igual modo, resulta ser muy elevada la necesidad manifestada por el alumnado que asiste a programas educativos de autorrealización y de superación personal (Fernández Lópiz, 2002, p. 168-175; Yuni, 1999).

Siguiendo este hilo y considerando ideas grupoanalíticas, apuntamos ahora que para constituir un grupo se precisan al menos cinco condiciones mínimas: un convocador, la presencia estable de unas personas, normas mínimas higienizadoras, el uso de la palabra y un referente teórico (Sunyer, 2001; Fernández Lópiz, 2002, p. 178-182). Detengámonos un poco en estas ideas y analicemos estas condiciones que en realidad son funciones psíquicas:

a. Función convocante. Alude a un conjunto de operaciones mentales por las que cada una de las vertientes de esta función, la convocada y la convocante, deben estar presentes y activas. Mientras que la convocante reside mayormente en el educador-facilitador, la función de ser convocado reside en el alumnado. Esta función mental es la responsable de que el deseo de organizar un grupo o de participar en él, pueda desarrollarse y concretarse. Como decimos, son responsables de esta función, tanto educadores como alumnado. Pero no siempre es así. En ocasiones existe una apreciable distancia entre la persona responsable del grupo y las 
personas que asisten a él, lo cual es fuente de problemas diversos. La importancia de este punto es crucial, pues la sintonía educador-alumnado mayor es más importante, que si de alumnado joven o infantil se tratara. Entre el facilitador y los convocados mayores ha de haber armonía y una buena relación, sintonía y congruencia. El facilitador debe proyectar un deseo, debe poder fantasear lo que desea que el grupo sea, lo cual atañe a las relaciones interpersonales que se establecen entre los miembros del grupo y que condicionan y son condicionadas, a su vez, no sólo por las características personales de los componentes del mismo, sino por las que se derivan de la actuación del facilitador.

En este punto quiero señalar que hay una serie de subfunciones que acompañan a la función convocante:

i. Unas de ellas son de carácter administrativo. Por ejemplo, la determinación de cuánta gente formará el grupo educativo; si el grupo es abierto o cerrado; si debe poner su acento en el debate científico o puede incorporar otras cuestiones, etc. Este aspecto reside en el educador.

ii. Otras son de proceso. Establecer los objetivos del grupo, es decir, las metas que se fijarán los reunidos. Este aspecto reside en el educador y en el alumnado.

iii. Un tercer tipo atañe a la sub-función grupal. Es decir, la función como proceso psicológico, mental, que determina la posibilidad de que el grupo se constituya como tal. Esta función reside originariamente en el educador. Otro de los términos de esta sub-función grupal es la de conductriz, que es la responsable de la articulación creativa de las fuerzas pro-grupales y las anti-grupales donde se encuentran los aspectos destructivos del grupo que tratan de impedir su proceso creativo. Esta sub-función reside originariamente en el profesor y se desplaza, posteriormente, a los miembros del grupo.

b. Función presencial. La presencia estable de los componentes. Es precisa la presencia activa de educador y alumnado, de forma que todos puedan reconocerse como miembros integrantes del grupo de clase. Esta cualidad hace que en este tipo de encuadre la ausencia de uno o varios de sus miembros altere la constitución del grupo docente-facilitador. De hecho, en los grupos educativos de mayores, los componentes suelen disponer unos mínimos niveles de compromiso entre ellos y el conjunto de la institución desde donde se organiza la actividad (Centro de Día, Residencia o Aula de Mayores de la Universidad); esto posibilita la comprensión y la búsqueda de significados a los sucesos cotidianos y a las relaciones que ahí surgen. El educador ha de reparar en la importancia de la función presencial, ya que sin ella, es muy difícil la posibilidad de comprensión o el establecimiento de significados compartidos. Esta función reside, a partes iguales, en el docente y en el alumnado. 
Sobre la Formación del Docente-Facilitador de Personas Mayores

c. Función higienizante. Afecta a la organización y la vertebración de la actividad: la concreción de comportamientos adecuados, la existencia de horarios, la asignación de espacios, articulación de reuniones, intereses comunes, $u$ otros aspectos de tipo administrativo. En otro plano, más latente, esta función se refiere y afecta a la fiabilidad de las relaciones, a la confidencialidad. Esta función, que es responsabilidad de todos los miembros del grupo educativo, conforma una serie de operaciones mentales por las que los componentes del mismo se preocupan de aspectos saludables: es la matriz del grupo.

d. Función verbalizante. Esta función sirve como medio para incrementar la comprensión de los fenómenos que se dan, y para articularlos. Es decir, la palabra y en ocasiones el lenguaje no verbal, articulan las relaciones que se dan entre los miembros en el aula. No obstante, siempre preferiremos que nuestro alumnado pueda verbalizar el lenguaje no verbal, lo que posibilita que éste adquiera una dimensión más elaborada, más articulada, incluso con dimensiones ocultas que pueden aparecer en el aula en forma de afanes, deseos, temores o recursos defensivos como el silencio o la no participación. La característica de esta función reside básicamente en el "facilitador", y sólo posteriormente, también en el alumnado.

e. Función teorizante. Su referente se encuentra en la estructura pedagógica del grupo y en último término debe estar en la mente del educador. Se trata de la ideología del grupo (sin que deba confundirse con la filosofía que inspira los idearios del centro o institución desde donde se organiza la actividad o los objetivos que se definen en los estatutos del establecimiento en cuestión). Este referente teórico es el articulador que posibilita la comprensión de los fenómenos psicológicos que surgen de las relaciones entre los miembros del alumnado. Es el marco de pensamiento psicológico que nos permite a los educadores de mayores una mejor comprensión de los fenómenos, de la comunicación y de las relaciones que se generan en el aula. Esta función reside en el educador.

\section{Comunidad Educativa y Psicología Dinámica}

Es común abordar el trabajo educativo con mayores desde dos posicionamientos igualmente erróneos: uno que considera que dicha situación pedagógica es equivalente a la que se realiza con alumnado joven, lo que conlleva un desconocimiento de las particularidades de este tipo de alumnado, tal como venimos señalando. Y otra que considera la educación en las edades avanzadas como un tipo de "educación especial” (para disminuidos), con lo cual dichas propuestas carecen de consistencia para producir aprendizajes y avances en el desarrollo personal. Recordamos en este punto el peligro de la emergencia de prejui- 
cios de carácter “viejista” (Burba; Fernández Lópiz; González de Menne; Luque, 2002; Fernández Lópiz, 2012a, p. 17-19).

Es necesario subrayar que la actividad educativa con mayores reviste algunas particularidades, acorde al tipo de alumnado. La educación con personas mayores supone ritmos de aprendizaje diferentes, motivaciones distintas, un mayor nivel de individuación del sujeto, una capacidad para apropiarse de sistemas simbólicos ya experimentados, y un nivel distinto de autodesarrollo y de trascender la propia mismidad para focalizar afanes e intereses en colectivos más amplios: la familia, el barrio, la ciudad, el mundo en definitiva. Esta edad difiere de otras edades y sus particularidades plantean al educador un abordaje para el que se han sugerido criterios de intervención socio-educativa que incluyen una mayor implicación emocional (Tamer, 1995).

A continuación señalamos sucintamente los fundamentos teóricos e interventivos basados en una psicología dinámica. Este enfoque implica criterios y asunciones en los que inspirar la tarea docente, entre ellos:

- Actitud de comprensión del rol de los mayores en el ámbito educativo y en la sociedad en general. Hay en ello una fundamento en conocidas como las de Carl Rogers o Viktor Frankl.

- Hipótesis grupoanalíticas y dinámicas sobre la formación del Equipo Docente.

A estos planteamientos deben agregarse los de la Ecología Social iniciada por Moos y Lemke (1994), que entiende que toda organización social genera un Clima Social con personalidad propia, que le confiere unidad y coherencia. Desde esta perspectiva, habría que considerar ambientes socio-educativos más positivos que otros en relación a variables de carácter psicosocial como la complementariedad comunicativa entre el alumnado y entre estos y el docente, el manejo correcto de parte de los facilitadores de los refuerzos sociales, la formación del personal en el abordaje de conflictos, etc. (Fernández Lópiz, 2000a).

Para el logro de organizaciones educativas con estas características es necesario implementar una capacitación del equipo de educadores. En esta formación psico-socio-educativa debe ser ofertada por los Centros e Instituciones que brindan este servicio. Esta formación ha de perseguir una mejora en el clima social de la organización educativa en su conjunto. Y es que la órbita relacional en el aula está, obviamente, muy determinada por los estilos educativos. Así, la estabilidad y la armonía socio-afectiva entre los miembros del equipo docente, como la que debiera haber entre estos y los alumnos mayores, son un factor de cambio en el clima social dentro del aula o de la propia institución que organiza la actividad. 


\section{La Formación del Equipo Docente con Técnicas de Análisis Transaccional}

Dada la complejidad del fenómeno del envejecimiento y la multiplicidad de facetas implicadas en el diseño de intervenciones dirigidas a personas mayores, la formación de los educadores requiere de múltiples enfoques y el concurso de varias disciplinas (Didáctica, Psicología, Gerontología, etc.). Hecho este reconocimiento, mostramos ahora una propuesta de formación psicológica. Debido a nuestra experiencia contrastada en ocasiones diversas (Fernández Lópiz, 2000b, p. 209-217), proponemos la herramienta del Análisis Transaccional. Se trata de una técnica que ahorra tiempo y resulta atractiva, a la vez que resulta terapéuticamente eficaz, dado que permite la comprensión y la modificación de las conductas desajustadas.

La estructura social de una organización educativa y el estilo educativo de la misma, tiene una definitiva relación con los beneficios irradiados sobre el alumnado. Cabe entonces hacer algunas consideraciones sobre este tipo de organizaciones:

- Deben tener un efecto beneficioso, optimizador, sobre el alumnado.

- Han de servir al apoyo y vitalización del trato individual y grupal.

- Se ha de perseguir ofrecer oportunidades reales para la participación: democratización y toma de decisiones por parte del alumnado (Bedmar; Fresneda; Muñoz, 2004).

- Las actividades educativas con mayores son potencialmente terapéuticas.

- Toda organización crea un ambiente propio, una cultura determinada definida por el cuantum de "tolerancia" reinante.

- Finalmente, destacamos el valor concedido a la comunicación, que debe ser buscada y facilitada por todos los medios posibles.

\section{Líneas para un Programa de Formación con Técnicas de Análisis Transaccional}

El motivo del programa está expuesto en toda su extensión en Fernández Lópiz (2000b) y es su función formar a los facilitadores-educadores con el fin de mejorar su actuación educativa. Para ello hay que dotar al educador de herramientas para analizar su actuación docente, así como su capacidad de comunicación (transacciones) en el aula. Además, para cumplir los objetivos de mejora, se establecen "contratos terapéuticos” con los docentes asistentes, que son revisados por el coordinador del curso en el transcurso de varias semanas.

Los objetivos del Programa son: 
1) Dar a conocer la teoría y las técnicas del Análisis Transaccional y sus aplicaciones prácticas en las áreas de:

a) El análisis del funcionamiento personal: capacidad para analizar conductas propias y ajenas, y competencia para detectar anomalías en la función como educador.

b) El análisis de la relación socio-educativa con los mayores, con especial énfasis en el abordaje de conflictos y problemáticas diversas.

c) Aprendizaje de habilidades sociales: manejo de refuerzos sociales.

d) Cambio de actitudes y posicionamientos personales sobre la población mayor: prejuicios o juicios poco fundados, actitudes "viejistas" (Fernández Lópiz, 2003a).

2) Fomento de la complementariedad educador- alumnado mayor.

3) Orientar todo lo anterior a la potenciación de la comunicación y el logro de la cohesión interpersonal.

En lo que toca a los ejes conceptuales sobre los que se centra el Programa, estos son:

- Fundamentos y bases del Análisis Transaccional: bases teóricas y prácticas del A.T.

- Estructura y funcionamiento de la personalidad del docente: Estados del Yo, complementariedad y aplicabilidad de los conocimientos.

- Las transacciones (comunicación) en el aula. Comunicaciones complementarias, cruzadas y ulteriores o de doble sentido.

- Los mensajes en la relación educativa y su importancia en el mantenimiento de una atmósfera grupal óptima. Mensajes de crecimiento vs. Mensajes obstructores.

- Análisis de actitudes ante el alumnado mayor.

- Teoría de las caricias: reconocimiento y reforzadores sociales en el quehacer educativo.

- Aportaciones a la solución de conflictos en el aula. Los Juegos Psicológicos.

- La motivación en la labor como educadores de adultos mayores. Importancia de las necesidades egóticas y de autorrealización.

- Formas de organización del tiempo.

- Contratos terapéuticos. Objetivos a alcanzar.

Esta propuesta sintética de formación con los docentes permite abordar las complejas facetas del trabajo educativo. La optimización de la tarea y la organización educativa, debe ser contrastada para valorar su impacto sobre los resultados y las condiciones de aprendizaje

Educação \& Realidade, Porto Alegre, v. 40, n. 1, p. 149-171, jan./mar. 2015. 165 
Sobre la Formación del Docente-Facilitador de Personas Mayores

del alumnado mayor: empleo de herramientas de evaluación, escalas, observación y entrevistas con facilitadores y alumnado transcurrido un tiempo tras la implementación del programa.

\section{Efectos de la Educación sobre el Alumnado Mayor: mejoras e incidencias}

El alumnado mayor percibe que su experiencia favorece cambios y mejoras en su desarrollo personal. Los resultados obtenidos en un estudio dirigido por nosotros (Yuni, 1999) son claros en este sentido. Según esta investigación, el valor medio de cambio fue percibido por las personas mayores asistentes a programas educativos como "cambios importantes". Se evaluó el efecto de la educación sobre distintas dimensiones y los datos revelaron un efecto diferencial: mayores cambios en ciertas dimensiones y mejoras menos notables en otras. Describimos los resultados sucintamente.

- Autopercepción. Las mejoras más importantes se relacionaron con modificaciones en el sistema de auto-representaciones, formas de percibirse a sí mismos como sujetos sociales con posibilidades intelectuales y de aprendizaje. El alumnado mayor consideró, entre los principales beneficios de la educación, aquellos relacionados con la posibilidad de elaborar una nueva imagen interna.

- Favorecedor de intereses. Un segundo grupo de aspectos mejorados en este estudio fue la canalización de intereses y actividades mantenidas durante las etapas anteriores de la vida. La educación facilitó la concreción de deseos y expectativas a las que los sujetos no habían podido acceder previamente.

- Estilo de vida. El alumnado percibió también mejoras en el estilo y calidad de vida personal, como consecuencia de la adquisición de nuevos hábitos de cuidado y de ocupación del tiempo libre, y por una mejora en la comprensión de las características del entorno socio-cultural, lo que va acompañado de una reducción de temores y de un aumento de la seguridad personal.

- Género y nivel de educación. Se pudo apreciar también en esta investigación modificaciones en la percepción global de mejora con relación a dos variables: género y nivel educativo.

- Nivel educativo. Las personas con menores recursos educativos tendieron a valorar de manera más positiva la contribución de la educación en su proceso de desarrollo. Para las personas mayores con mejor nivel educativo la magnitud del cambio percibido fue menor. Esto puede explicarse porque aquellos que poseen mayores recursos cognitivos e informativos, tienden naturalmente a mantenerlos e incrementarlos, además de que en sí mismo, este nivel más elevado facilita a quienes lo poseen el acceso a otras fuentes de información y posibilita el tránsito a otro tipo de actividades de participación e integración social, a diferencia de quienes no lo poseen. Se hace evidente así, el papel que 
puede desempeñar la educación con personas mayores para evitar la deprivación cultural, tanto en cuanto al acceso a los bienes educativos y formativos, como a la posibilidad de que las desigualdades sociales de antaño, no sean un impedimento, en el caso de que sean las instituciones públicas quienes oferten esta posibilidad.

- Género. La muestra de este estudio, es preciso decirlo, era eminentemente femenina. En líneas generales, las mujeres informaron de un mayor cambio que los varones, y en un análisis más fino, se destaca que las diferencias son significativas en integración social y en mejoras metacognitivas. Ello muestra el papel que puede tener la educación de cara a la integración de las mujeres en nuevos grupos sociales, abriendo el círculo de interacción y rompiendo el aislamiento y la invisibilidad. Puede interpretarse también que la educación constituye un importante recurso para la reelaboración de una identidad como mujer mayor, a partir de la posibilidad de construir un proyecto de desarrollo personal propio, no definido exclusivamente por el cumplimiento de los tradicionales roles de género: esposa, madre, abuela, ama de casa, doméstica perenne, etc. En cuanto a la dimensión metacognitiva, o sea, la conciencia que una persona tiene de sus capacidades y habilidades cognitivas, también las mujeres obtuvieron más provecho de la educación. La explicación está, sobre todo, en variables generacionales, dado que este alumnado femenino proviene de una generación en que la mujer tuvo dificultades para acceder a la cultura.

- Confluencia de factores. En esta investigación se demuestra una interrelación de factores causales (explicativos). Como quiera que se observaran elevadas correlaciones entre diferentes dimensiones consideradas, este aspecto revela el carácter interactivo de ellas y sugiere su interdependencia y potenciación recíproca. Los efectos que la educación precipita en los mayores son muy variados y están íntimamente conectados.

- Educación y metas de intervención. El carácter complejo e interdependiente de los factores involucrados en la educación, está en la base de la mejora que reflejan las diferentes dimensiones evaluadas. De este modo, se pone de relieve que la intervención educativa es altamente eficaz para operar simultáneamente sobre dimensiones diversas como las representacionales, las cognitivas, las relacionales, las actitudinales, etc. Frente a las mejoras parciales que producen los programas de entrenamiento cognitivo concretos, la educación implica la interacción con otros, el uso de símbolos comunes, la confrontación con puntos de vista distintos, el acceso al mundo de la información, la adquisición de medios intelectuales, todo lo cual precipita una amplia y variada movilización de recursos y se manifiesta en una mejora que comprende diversas dimensiones a la vez.

Esta investigación confirma que la educación con personas mayores es pertinente para plantear objetivos polivalentes de intervención, considerando los múltiples condicionamientos personales, sociales y 
Sobre la Formación del Docente-Facilitador de Personas Mayores

culturales que particularizan las necesidades, posibilidades y obstáculos para el desarrollo de las personas de edad.

\section{Conclusiones Finales}

La formación de los educadores constituye un tema poco investigado en la literatura educativa. Esta falta de reflexión e indagación sugiere la presencia de un fenómeno de empirismo profesional, toda vez supone que se aprende a ser educador de mayores sobre el terreno, sin que haya apenas especialización en este ámbito tan pujante hoy día. Hemos querido poner de manifiesto en estas líneas, la necesidad formativa del educador cuya labor se va a ejercitar con personas de edad, haciendo hincapié, no sólo en su dimensión intelectual o de aprendizaje, sino también en el carácter socio-afectivo de este tipo de actividad (Fernández Lópiz, 2012b).

Además, la educación con personas de edad se produce en un espacio compartido, pero a la vez propio. Es una necesidad de los mayores pasar de la dependencia a la independencia, del estancamiento indeseable a una manera de actividad y empleo ilusionante y creativo de su tiempo. El educador debe promover que las personas mayores alcancen un grado de competencia como protagonistas sociales, que incorporen esa dimensión protagonista a la propia mismidad. Los mayores necesitan capacitarse por medio de la educación, para otra manera de percibir las relaciones entre ellos y el mundo. Eso hará que sientan más confianza en su habilidad para enfrentarse a una sociedad cambiante y a sus propios problemas, con más seguridad, más serenamente. Lo que sucede entonces es que el sujeto estará dominando el material bruto de su propia experiencia, comenzando a aceptar que esta es una parte que contribuye de forma importante a la experiencia modelada por la sociedad como un todo. Y, sin duda, la educación es un medio para conseguir estos objetivos (Fernández Lópiz; Ferreiro, 2006).

Recibido en 10 de marzo del 2014 Aprobado en 28 de julio del 2014

\section{Nota}

1 Según Winnicott (1981), el análisis de las personas sugiere que entre el individuo y la realidad existe una zona intermedia y neutral de experimentación, a la cual contribuyen tanto la realidad interior como la vida exterior. Es una zona que no es disputada, ya que ninguna reivindicación se hace por cuenta de la misma, salvo la de que exista como lugar de descanso para el individuo metido en la perpetua tarea humana de mantener separadas, y a la vez interrelacionadas, la realidad interior y la exterior. Winnicott (1981, p. 315) afirma, que la "puesta a prueba de la realidad" no es fácil. Entonces, el psiquismo lo que hace es reclamar la existencia de un estado intermedio para reconocer y aceptar la realidad de una forma más sosegada. Da Winnicott (1981, p. 328) “[...] por senta-

168 Educação \& Realidade, Porto Alegre, v. 40, n. 1, p. 149-171, jan./mar. 2015. Disponível em: <http://www.ufrgs.br/edu_realidade> 
do que la tarea de la aceptación de la realidad jamás es completada, que ningún ser humano está libre de la tensión que ocasiona el relacionar la realidad interior con la exterior y que el alivio de tal tensión lo aporta una zona intermedia de experiencias que no es disputada (el arte, la religión, etc.) [...] Esta zona intermedia se halla en continuidad directa con respecto a la zona de juegos donde el niño pequeño se 'pierde' al jugar". Es por esto que aparecen, primero en los niños, los llamados objetos transicionales en forma de un osito de peluche o cualquier objeto preferente que sin ser el niño (Objeto No Yo) ni la madre (Objeto No madre), conforman ese espacio limítrofe (ilusionante) que le tranquiliza sobre todo ante la "angustia de separación". Winnicott estudia en este punto la sustancia de la ilusión, aquello que le es permitido al pequeño y que, luego, en la vida adulta, es inherente al arte, a la religión, a la ciencia, al amor o a tantas ilusiones que albergamos de mayores y sin las cuales la vida se haría muy costosa. Por eso, Winnicott no es que se refiera exactamente al osito de trapo del pequeño ni al uso inicial que éste hace de esos objetos, sino a la zona intermedia que hay entre lo subjetivo y lo que es percibido objetivamente. Resumiendo, estos objetos conforman lo que Winnicott denominó "el espacio de lo ilusorio", que es un espacio psíquico, origen de las ilusiones. A lo largo de la vida los fenómenos transicionales pierden su original significado y quedan extendidos por la esfera intermedia entre la realidad psíquica interna y el mundo exterior compuesto por el vasto terreno de las experiencias educativas, culturales, religiosas, la imaginación o la labor científica y creadora en general. Es aquí donde el aporte del autor se ensancha y amplía a otras edades. Lo que ocurre es que estos objetos antiguos, o mejor, ese espacio de lo ilusorio infantil se reconvierte en ilusiones más complejas: la educación, la sed de conocimiento, la necesidad de viajar, el afán de saber, la lectura, la afición al cine, etc. Por ello hablamos de "motivación ilusoria". El espacio educativo, que es un espacio compartido, pero a la vez propio, permite el tránsito de la dependencia a la independencia, del estancamiento a una manera de actividad y empleo ilusionante, creativo y compartido del tiempo.

\section{Referencias}

BALTES, Paul; SMITH, John. Psicología de la Sabiduría y su Ontogénesis. In: STERNBERG, Robert J.: La Sabiduría: su naturaleza, orígenes y desarrollo. Bilbao: Desclée de Brouwer, 1994. P. 109-147.

BEAUVOIR, Simone de. La Vejez. Barcelona: Edhasa, 1983.

BEDMAR, Matías; FRESNEDA, Ma Dolores; MUÑOZ, Juana. Gerontagogía: educación en personas mayores. Granada: Editorial de la Universidad de Granada, 2004.

BURBA, María Cristina; FERNÁNDEZ LÓPIZ, Enrique; GONZÁLEZ VERHEUST, María Cristina; LUQUE, Leticia Elizabeth. Vejez y Diversidad. Córdoba (Argentina): Editorial Brujas, 2013.

BURBA, María Cristina; FERNÁNDEZ LÓPIZ, Enrique; GONZÁLEZ DE MENNE, María Carmen; LUQUE, Leticia Elizabeth. Discriminación por Viejismo en Estudiantes Universitarios: un estudio transcultural”. Psico-Logos, Universidad Nacional de Tucumán, p. 104-116, oct. 2002.

CALERO, María Dolores; LOZANO, María Ángeles. La Evaluación del Potencial de Aprendizaje en Ancianos. Revista de Psicología General y Aplicada, v. 47, n. 1, p. 89-100, 1994.

Educação \& Realidade, Porto Alegre, v. 40, n. 1, p. 149-171, jan./mar. 2015. 
Sobre la Formación del Docente-Facilitador de Personas Mayores

CLAYTON, Vivian. Wisdom and Intelligence: the nature and function of knowledge in the later years. International Journal of Aging and Human Development v. 15, p. 315-321, 1982.

DITTMAN-KOHLI, Freya. Aspects of Cognitive Development in Adulthood. In: II CONFERENCIA SOBRE PSICOLOGÍA DEL DESARROLLO. Actas... Roma, Italia, 1986. P. 145-161.

FERNÁNDEZ BALLESTEROS, Rocío et al. Evaluación e Intervención Psicológica en la Vejez. Barcelona: Martínez Roca, 1992.

FERNÁNDEZ LÓPIZ, Enrique. Ideas y Actitudes Improductivas en la Enseñanza con Alumnos Mayores. Propuestas: Revista de la Universidad Nacional de la Matanza, Año III, n. 6, p. 107-128, Buenos Aires (Rep. Argentina), 1997

FERNÁNDEZ LÓPIZ, Enrique. Psicogerontología: perspectivas teóricas y cambios en la vejez. Granada: Adhara Ediciones, 1998a.

FERNÁNDEZ LÓPIZ, Enrique. Ilusión, Identidad y Educación en las Personas Mayores: una apertura a la experiencia. Talis: Bulletin of the Talis Network, $n$. 8, p. 93-105, 1998b.

FERNÁNDEZ LÓPIZ, Enrique. Explicaciones sobre el Desarrollo Humano. Madrid: Pirámide, 2000a.

FERNÁNDEZ LÓPIZ, Enrique. Los Equipos Asistenciales en las Instituciones: guía teórico-práctica de técnicas de análisis transaccional. Madrid: Narcea, 2000b.

FERNÁNDEZ LÓPIZ, Enrique. La Psicogerontología y su aplicación al terreno de la educación con mayores. In: VICENTE, Pedro de; MOLINA, Enriqueta (Coord.). Salidas Profesionales de los Estudiantes de Pedagogía. Un reto para el Prácticum. Facultad de Ciencias de la Educación: Universidad de Granada, 2001. P. 185-199.

FERNÁNDEZ LÓPIZ, Enrique. Psicogerontología para Educadores. Granada: Editorial de la Universidad de Granada, 2002.

FERNÁNDEZ LÓPIZ, Enrique. Mitos Improductivos en la Enseñanza con Alumnos Mayores. In: CARRERAS, Juan Sáez (Coord.). Educación y Aprendizaje en las Personas Mayores. Madrid: Editorial Dykinson, 2003a. P. 209-230.

FERNÁNDEZ LÓPIZ, Enrique. Psicogerontología para Educadores. In: PÉTRIZ, Graciela (Coord.). Nuevas Dimensiones del Envejecer: teorizaciones desde la práctica. Edita Programa Permanente de Adultos Mayores (PEPAM). Facultad de Humanidades y Ciencias de la Educación. Universidad Nacional de La Plata, 2003b. P. 33-64.

FERNÁNDEZ LÓPIZ, Enrique. Psicología del Envejecimiento. Granada: Grupo Editorial Universitario, 2012a.

FERNÁNDEZ LÓPIZ, Enrique. Sobre la Necesidad de la Materia de Psicogerontología en las Facultades de Ciencias de la Educación. Revista de Análisis Transaccional y Psicología Humanista, n. 67, Año XXX, p. 218-234, 2º Semestre/2012, 2012b.

FERNÁNDEZ LÓPIZ, Enrique. Necesidad de Formación en Psicogerontología y Educación con Mayores para el Voluntariado: ideas y reflexiones. In: ALARCÓN, Vicente Ballesteros (Coord.). Voluntariado y Personas Mayores. Investigaciones y Experiencias. Editorial Universidad de Granada, 2013. Capítulo 2, p. 22-29 (Libro en formato Cd ROM).

FERNÁNDEZ LÓPIZ, Enrique; YUNI, José Alberto. Ilusión, Identidad y Educación en las Personas Mayores: una apertura a la experiencia. Annual Bulletin 
of the TALIS Network, Third Age Learning International Studies, n. 8, p. 93-105, University of Toulouse (Francia), 1998.

FERNÁNDEZ LÓPIZ, Enrique; MARÍN PARRA, Vicenta; ALFARO LLANEZA, Isabel. Género y Envejecimiento Humano. Revista Conceptos: Boletín de la Universidad del Museo Social Argentino, n. 3, p. 36-42, Buenos Aires (Rep. Argentina), Mayo/Junio 1999.

FERNÁNDEZ LÓPIZ, Enrique; FERREIRO, Ma Teresa: El Sentido de la Vejez en el Tiempo de Hoy. Geriátrika: Revista Iberoamericana de Geriatría y Gerontología, v. 22, n. 6, p. 43-51, 2006.

MARTÍN GARCÍA, Antonio Víctor. Educación y Envejecimiento. Barcelona: PPU, 1994.

MOOS, Rudolf; LEMKE, Sonne. Group Residences for Older Adults: physical features, policies, and social climate. New York: Oxford University Press, 1994.

NAVAL, Concepción. La Participación Social de las Personas Mayores. In: ORDUNA, Gabriela; NAVAL, Concepción (Ed.). Gerontología Educativa. Barcelona: Ariel Educación, 2001. P. 123-156.

STERNBERG, Robert J. Cognitive Approaches to Intelligence. In: WOLMAN, Benjamin B. (Ed.). Handbook of Human Intelligence: theories, measurements and applications. New York: Wiley Intercience, 1985. P. 59-118.

SUNYER, Mikel. Reflexiones en torno a un Espacio Singular: el seminario. Boletín: Revista de la Asociación de Psicoterapia Analítica Grupal, n. 24, p. 3-17, 2001.

TAMER, Norma. El Envejecimiento Humano. Sus derivaciones pedagógicas. Washington: Colección Interamer (Organización de los Estados Americanos), 1995.

WINNICOTT, Donald. Objetos y Fenómenos Transicionales. Estudio de la primera posesión `No Yo`. In: WINNICOTT, Donald (Org.). Escritos de Pediatría y Psicoanálisis. Barcelona: Ed. Laia, 1981. P. 313-330. (1ª Ed. Londres, 1958).

YUNI, José Alberto. La Motivación Académica en la Segunda Mitad de la Vida. Trabajo de Investigación del Programa de Doctorado: intervención psicológica en contextos educativos y de desarrollo. Departamento de Psicología Evolutiva y de la Educación, Universidad de Granada, España, 1997. (Director: Dr. Enrique Fernández Lópiz).

YUNI, José Alberto. Optimización del Desarrollo Personal mediante la Intervención Educativa en la Adultez y la Vejez. Tesis Doctoral, Departamento de Psicología Evolutiva y de la Educación. Universidad de Granada, España, 1999. (Director: Dr. Enrique Fernández Lópiz).

Enrique Fernández Lópiz cursó la Licenciatura de Psicología en la Universidad de Salamanca y obtuvo su Doctorado en Psicología por la Universidad de Granada. Es psicoterapeuta, miembro de la Federación Europea de Asociaciones Psicoterapeutas (FEAP). En el terreno académico, es Profesor Titular del Departamento de Psicología Evolutiva y de la Educación de la Universidad de Granada. Ha impartido clases y seminarios en diferentes Universidades españolas y extranjeras, y tiene en su haber una producción científica reflejada en revistas especializadas y en libros sobre Clínica y Psicoterapia, Psicología del Desarrollo y Psicogerontología.

E-mail: elopiz@ugr.es

Educação \& Realidade, Porto Alegre, v. 40, n. 1, p. 149-171, jan./mar. 2015.

Disponível em: <http://www.ufrgs.br/edu_realidade> 